\title{
Special announcement: Guidelines to the Practice of Anesthesia-Revised Edition 2020
}

\author{
Gregory R. Dobson, MD
}

Received: 2 September 2019/Revised: 2 September 2019/Accepted: 9 October 2019/Published online: 29 October 2019

(C) Canadian Anesthesiologists' Society 2019

This issue of the Journal features the 2020 revised edition of the Guidelines to the Practice of Anesthesia ${ }^{1}$ as developed and updated annually by the Canadian Anesthesiologists' Society (CAS) Committee on Standards and approved by the CAS Board of Directors. It represents the $43^{\text {rd }}$ annual revision of the Guidelines since they were first issued in 1977 and the $11^{\text {th }}$ year that they have appeared in the Journal itself. They are intended to provide a broad framework for safe anesthesia practice in Canada, wherever it is delivered.

It is ever more challenging for physicians to stay up to date with all of the medical literature related to healthcare delivery. Clinical practice guidelines contain recommendations that are developed from systematic review and critique of the available literature. They contain suggestions for approaches to the organization and delivery of care but should not be viewed as rigid, fixed standards that must be followed in every clinical situation (unless specified as such in the recommendation). The advice that they provide can range from the relatively discretionary to the very specific. Importantly, individual physicians should still feel confident that their knowledge, experience, and discretion is essential to the delivery of appropriate care. ${ }^{2}$

The Committee on Standards welcomes questions, comments, and constructive criticism related to the Guidelines. All submitted queries and their related responses are available on the CAS website. The commentary in this editorial should be considered an invitation to any anesthesiologist, allied health

G. R. Dobson, MD ( $₫)$

Canadian Anesthesiologists' Society, 1 Eglinton Avenue East,

Suite 208, Toronto, ON M4P 3A1, Canada

e-mail: standards@cas.ca professional, or healthcare administrator to report their questions or comments to the Committee on Standards (standards@cas) related to existing guideline content or topics that readers feel should be addressed in the Guidelines.

In light of the comprehensive and lengthy nature of the Guidelines document, this editorial serves to draw the reader's attention to specific updates in the new Guidelines and the evidence supporting these changes. It also provides commentary on issues that are under consideration for publication in future versions.

Appendices to the Guidelines, position papers, and other guideline documents are regularly reviewed by the Committee and updated as required. To coincide with the release of the 2020 revised edition, in cooperation with the Canadian Standards Association Group, we are excited to have recently released an updated version of Appendix 1 Canadian Standards Association Standards for Medical Devices and Equipment. ${ }^{3}$ Appendix 4 - Guidelines Standards and Other Official Statements ${ }^{4}$ has been reorganized into a more user-friendly, topic-based format with clickable links that direct you to the relevant web resources. We are confident readers will find it much more useful and we plan to more regularly review and update the resources that are referenced. The Committee on Standards collaborates with the CAS section heads when considering areas that need improvement or updating the Guidelines.

The Committee actively considered a variety of topics for revision and implemented a number of changes that are highlighted in bold in the revised edition of the Guidelines. The following changes are amongst those implemented and they appear in italics below:

- In the 2019 edition ${ }^{5}$ under section 3.1-4 Responsibilities of the Healthcare Facility, the 
Committee introduced content related to the safe handling, storage, and disposal of controlled medications (e.g., opioids) to minimize the risk of diversion. For 2020, we have strengthened the recommendations by including specific examples of strategies to prevent diversion, based upon best practice. The new content reads: "Best practice principles support a variety of specific strategies to achieve these goals. The CAS does not endorse any one specific strategy or policy but strongly recommends that all available options be considered. Robust systems and procedures must be implemented. Due consideration should be given to any limitation in resources available to implement specific security systems and procedures within a facility, particularly for smaller facilities.

Recommendations include, but need not be restricted to:

- storing controlled substances in a secure lockable safe or locked drawer when the operating room is unattended by anesthesia personnel (e.g., between cases);

- never leaving controlled substances (including those drawn up into syringes or bags) unattended in any location including in the operating room;

- emptying the contents of syringes and bags containing controlled substances before disposal;

- adopting disposal and/or destruction systems to dispose of controlled substance waste;

- consideration of a requirement that controlled substance waste be returned to the pharmacy and be subjected to random analysis;

- periodic audits of a healthcare providers' controlled substance utilization records and their anesthesia records;

- consideration of the implementation of automated anesthesia cabinets or automated medication dispensing cabinets (e.g., Pyxis $\left.{ }^{T M}\right)$.

The operating room, labour and delivery, postanesthesia care unit, and regional block areas, for example, should all be recognized as high-risk areas for controlled medication diversion and therefore must be subject to heightened security, surveillance, and auditing."

- Section 4.2 Fasting Guidelines has been updated. The Canadian Pediatric Anesthesia Society have made new recommendations related to clear fluid fasting for infants and children, which have been incorporated into the Guidelines. ${ }^{6}$ Premedication recommendations for elective and emergent Cesarean delivery have also been added. The updated fasting guidelines now read:

"Before elective procedures, the minimum duration of fasting should be:
- Eight hours after a large meal of solids particularly containing protein (e.g., meat) or fatty foods;

- Six hours after a light meal (e.g., non-fatty meal such as toast);

- Six hours after ingestion of infant formula, non-human milk, or expressed breast milk fortified with additions;

- Four hours after ingestion of breast milk;

- Two hours after ingestion of clear fluids for adults;

- One hour after ingestion of clear fluids for infants and children".

Recommendations for premedication before elective and emergent Cesarean delivery have been added as follows: "An oral $\mathrm{H} 2$ receptor antagonist is recommended the night before and on the morning of an elective Cesarean delivery.

An intravenous $\mathrm{H} 2$ receptor antagonist and $30 \mathrm{~mL}$ of oral 0.3 molar sodium citrate are recommended prior to an emergent Cesarean delivery if general anesthesia is planned"

In addition, under section 7.3 Oral Intake During Labour, it is now considered acceptable to permit women in active labour to ingest clear fluids as desired. Although opinion varies widely, and the evidence for any benefit or harm to parturients ingesting solid foods during active labour is limited, ${ }^{7,8}$ parturients should generally be discouraged from ingesting solid foods while in active labour. Nevertheless: "In contrast to solid food, clear fluids are emptied relatively rapidly from the stomach during labour. Therefore, in general, women should be permitted clear fluids as desired during active labour. Individual facilities should develop protocols regarding the intake of solids and clear fluids by women in active labour".

- Despite the steady improvement as a specialty, it is well recognized that airway management, particularly of the difficult airway, still contributes to a significant proportion of anesthesia-related adverse events. ${ }^{9}$ To date, the Guidelines have been silent related to airway management. The Committee has introduced a new section 5.2 Airway Management to make some initial recommendations. This section will be further enhanced in future editions of the Guidelines. It reads: "Airway management, particularly of the difficult airway, contributes to a significant proportion of anesthesia-related morbidity and mortality. The appropriate management of those patients who have a predicted or an unanticipated difficult intubation, a failed airway, where bag-mask ventilation or supraglottic device placement may be difficult, or who require a surgical airway is critical for patient safety. This includes, but need not be restricted to, adequate airway assessment, equipment (e.g., difficult airway carts, videolaryngoscopes, 
bronchoscopes), training and simulation, support personnel and the use of protocols and cognitive aids to optimize difficult airway management. The CAS does not endorse any one specific protocol, algorithm, or cognitive aid for difficult and failed airway management but strongly recommends that readers refer to Appendix 4 for up to date publications related to this topic."

- Cautious dosing, attention to detail, and the routine use of neuromuscular monitoring are well recognized components of safety and best practice when using neuromuscular blocking agents. The 2018 edition of the Guidelines introduced the recommendation that monitoring "should" be utilized. It is not controversial that monitoring is essential to accurately assess satisfactory neuromuscular recovery. The Committee felt that the recommendation should be strengthened and therefore: "Neuromuscular monitoring must be utilized when neuromuscular blocking agents are used". This author feels strongly that quantitative (objective) monitoring (e.g., electromyography, kinemyography, accelerometry) is ideal and superior to qualitative (subjective) monitoring with peripheral nerve stimulators using tactile and visual measurements. ${ }^{10-12}$ At this time, however, the Committee has chosen to simply recommend that monitoring be utilized, leaving the type of monitoring to the discretion of the attending anesthesiologist because quantitative monitors are not always available. Neuromuscular blockade monitors have also been moved from "exclusively available" to "required" monitoring equipment in section 5.5 Required Monitoring Equipment when neuromuscular blocking agents are used.

- Important changes have been made to section 5.7 Records. It is essential to document unexpected and adverse events during anesthesia care. Adverse event recording and reporting systems must be an important part of departmental quality and safety programs (see section 2.1-4) or departments can choose to report using national programs such as the CAS Canadian Anesthesia Incident Reporting System. ${ }^{13}$ In addition to recording monitored vital signs and routine events: "All other relevant intraoperative anesthesia care and events, including unexpected or adverse events, should also be recorded".

Electronic anesthesia information management systems (AIMS) have replaced manual handwritten anesthesia records in some centres where adequate resources have been available. The anesthesia patient safety literature strongly supports the use of AIMS. The Committee has introduced some recommendations related to the potential advantages of AIMS over handwritten records. Nevertheless, as AIMS systems are still maturing, they require a clinical champion to work with the information technology/biomedical team during implementation and use, and they can potentially represent a distraction. The potential benefits are felt to outweigh any limitations. ${ }^{14}$ The new content reads: "These recommendations apply to both manually created (handwritten) and electronic anesthesia information management system (AIMS) created anesthesia records. At present there are no practice standards for what additional data (e.g., gas analysis, ventilator, and respiratory data), that can potentially be collected by an AIMS charting system, should become part of a patient's permanent health record.

The AIMS have been shown to have potential benefits over handwritten records in several key areas, including improved legibility, precision and reliability of anesthesia records, providing searchable data for quality improvement, outcome and performance reporting and translational research, enhanced medication safety and tracking, and real-time clinical decision support for users. The anesthesia patient safety literature strongly supports the use of AIMS. Importantly, AIMS maintain a longitudinal patient database so historical patient encounters should be easily retrievable for review and this should be considered an important feature of any system. The CAS does not promote or endorse any one specific vendor or product but acknowledges that there may be potential benefits of a well-designed and implemented AIMS over manually charted records where facility and anesthesia department resources permit the consideration of an AIMS. Ideally it should be implemented and supported in cooperation with the facility information technology department including communication with other facility electronic patient databases wherever possible”.

The Standards Committee acknowledges that our process for guideline development could benefit from a more validated and systematic approach, for example, using the Grading of Recommendations Assessment, Development and Evaluation (GRADE) framework for rating the quality of evidence when developing our recommendations. ${ }^{15} \mathrm{We}$ are evaluating how we currently develop our guidelines and are actively looking into an improved, more evidence-based process.

At present, the CAS does not have a process to rigorously evaluate and potentially endorse anesthesia practice guidelines created by individuals and groups outside of the CAS. We are looking closely at the potential benefits for CAS members if we were to develop and implement such a process.

Other topics that the Standards Committee are actively considering for future editions of the Guidelines include 
anesthesia human resources (as we face a shortage of anesthesiologists), leave and retirement policies, and enhancement of section 2.3 Fitness to Practice as relates to the identification and support of anesthesia professionals who may be facing personal or health difficulties or may be impaired. In cooperation with the Committee on the Anesthesia Care team we are currently extensively revising Appendix 5-Position Paper on Anesthesia Assistants. We plan to release an updated document in the near future.

In closing, I would like to personally thank Dr. David McKnight and Dr. Hilary Grocott for their invaluable assistance and support of the Guidelines during my term as Standards chair.

\section{Communiqué spécial : Guide d'exercice de l'anesthésie - Édition révisée 2020}

Ce numéro du Journal présente l'édition révisée du Guide d'exercice de l'anesthésie ${ }^{1} 2020$, tel que conçu et annuellement mis à jour par le Comité des normes de la Société canadienne des anesthésiologistes (SCA), puis approuvé par le Conseil d'administration de la SCA. Il s'agit de la $43^{\mathrm{e}}$ révision annuelle du Guide depuis sa première édition en 1977 , et de sa $11^{\mathrm{e}}$ publication dans le Journal. L'objectif de ce Guide est de proposer un cadre général pour une pratique sécuritaire de l'anesthésie au Canada, peu importe où elle est pratiquée.

Il est de plus en plus difficile pour les médecins de rester à jour étant donné la quantité de littérature médicale s'intéressant à la fourniture des soins de santé. Les guides de pratique clinique comportent des recommandations mises au point à partir de revues systématiques et de critiques de la littérature disponible. Ils contiennent des suggestions d'approches à l'organisation et à la fourniture des soins. Toutefois, ils ne doivent pas être considérés comme des normes figées et rigides qui doivent être respectées dans toutes les situations cliniques (sauf mention spécifique dans le guide). Les conseils que ce Guide prodigue vont de recommandations relativement générales, laissées à la discrétion du fournisseur de soins de santé, à des directives très spécifiques. Il est important de souligner que les médecins devraient demeurer confiants en leurs connaissances, expérience et jugement, essentiels pour prodiguer des soins appropriés. ${ }^{2}$

Le Comité des normes reçoit volontiers toute question, tout commentaire, et toute critique constructive en ce qui touche au Guide. Toutes les questions soumises et les réponses associées sont disponibles sur le site Internet de la SCA. Le commentaire de cet éditorial doit être lu comme une invitation à tout anesthésiologiste, professionnel paramédical ou administrateur de soins de santé, à adresser ses questions ou commentaires au Comité des normes (standards@cas) en ce qui a trait au contenu du Guide existant ou à des thèmes qui mériteraient d'y être abordés.

Étant donné la nature exhaustive et la longueur du Guide, cet éditorial a pour but d'attirer l'attention du lecteur sur des mises à jour spécifiques dans la nouvelle version du Guide et aux données probantes justifiant ces modifications. Il sert également à présenter certains sujets que nous envisageons d'aborder dans de futures versions.

Les annexes du Guide, énoncés de position et autres documents de recommandations sont régulièrement révisés par le Comité et mis à jour au besoin. Afin de coïncider avec la publication de l'édition révisée de 2020, en coopération avec le Groupe de l'Association canadienne de normalisation, nous avons récemment publié une version mise à jour de l'Annexe 1 - Association canadienne de normalisation - Normes concernant le matériel. ${ }^{3}$ L'Annexe 4 - Lignes directrices, normes et énoncés généraux ${ }^{4}$ a été réorganisée dans un format plus convivial; les liens sont classés par thème et il est possible de cliquer directement sur ces liens afin d'accéder aux ressources internet pertinentes. Nous sommes confiants que les lecteurs trouveront ce document bien plus utile dans sa nouvelle mouture et avons l'intention de passer en revue et mettre à jour plus régulièrement les ressources mentionnées. Le Comité des normes collabore avec les chefs de section de la SCA pour évaluer les domaines à améliorer ou à mettre à jour dans le Guide.

Le Comité a examiné en détail plusieurs sujets pour les réviser et a mis en œuvre de nombreuses modifications, lesquelles sont surlignées en gras dans la version révisée du Guide. Vous trouverez en italique ci-dessous certaines modifications apportées :

- Dans la section 3.1-4 Responsabilités de l'établissement de santé, version 2019, ${ }^{5}$ le Comité avait introduit du contenu concernant la manutention, l'entreposage et l'élimination des médicaments contrôlés (par ex. les opioïdes) afin de minimiser le risque de détournement. Dans la version 2020, nous avons renforcé ces directives en incluant des exemples spécifiques de stratégies fondées sur les meilleures pratiques visant à éviter le détournement. Voici la nouvelle version: «Les principes des meilleures pratiques prônent diverses stratégies spécifiques pour atteindre ces objectifs. La SCA ne préconise pas une 
stratégie ou politique spécifique en particulier mais recommande fortement que toutes les options disponibles soient envisagées. Des systèmes et procédures fiables doivent être mis en ouvre. Une attention particulière doit être portée à toute restriction des ressources disponibles pour mettre en ouvre des systèmes et interventions de sécurité spécifiques au sein d'un établissement, particulièrement dans les plus petits établissements.

Les recommandations comprennent, sans nécessairement s'y limiter :

- l'entreposage des substances contrôlées dans un coffre-fort verrouillable et sécuritaire ou dans un tiroir verrouillé lorsqu'il n'y a pas de personnel d'anesthésie en SOP (par ex., entre deux cas);

- le fait de ne jamais laisser de substances contrôlées (y compris celles préparées dans des seringues ou des sacs) sans surveillance, quel que soit le lieu;

- la vidange du contenu des seringues et sacs contenant des substances contrôlées avant de les jeter;

- l'adoption de systèmes d'élimination et/ou de destruction pour se débarrasser des substances contrôlées inutilisées;

- une réflexion sur la possibilité d'exiger que les substances contrôlées inutilisées soient retournées à la pharmacie et soumises à des analyses aléatoires;

- des contrôles périodiques des dossiers d'utilisation des substances contrôlées par un professionnel des soins de santé et ses dossiers anesthésiques; et

- l'installation éventuelle d'armoires d'anesthésie automatisées ou d'armoires de distribution de médicaments automatisées (par ex. Pyxis ${ }^{T M}$ ).

Par exemple, les salles d'opération, d'accouchement, de réveil et de blocs régionaux devraient toutes être reconnues comme des zones à risque élevé de détournement de médicaments contrôlés; par conséquent, elles devraient faire l'objet d'une sécurité, d'une surveillance et de contrôles accrus. »

- La section 4.2 Lignes directrices concernant le jeûne a été mise à jour. La Société d'anesthésie pédiatrique canadienne a émis de nouvelles recommandations concernant le jeûne et l'ingestion de liquides clairs pour les nourrissons et les enfants, lesquelles ont été intégrées dans le Guide. ${ }^{6}$ Les recommandations de prémédication en cas d'accouchement par césarienne programmé et urgent ont également été ajoutées. Les lignes directrices concernant le jeûne ont été mises à jour comme suit :

«Avant une intervention non urgente, la durée minimale du jeûne devait être de :
- Huit heures après un repas copieux comportant des aliments solides, particulièrement s'il contenait des protéines (par ex. de la viande) ou des aliments gras;

- Six heures après un repas léger (par ex. repas faible en gras tel une tartine);

- Six heures après l'ingestion de lait maternisé, de lait non humain ou de lait maternel tiré et fortifié avec des adjuvants;

- Quatre heures après l'ingestion de lait maternel;

- Deux heures après l'ingestion de liquides clairs pour un adulte;

- Une heure après l'ingestion de liquides clairs pour les nourrissons ou les enfants. »

Les nouvelles recommandations de prémédication avant un accouchement par césarienne programmé ou urgent sont les suivantes : «Un antagoniste oral des récepteurs $\mathrm{H} 2$ de l'histamine est recommandé la nuit précédant et le matin d'un accouchement par césarienne programmé.

Un antagoniste intraveineux des récepteurs $\mathrm{H} 2$ de l'histamine et $30 \mathrm{~mL}$ de citrate de sodium molaire 0,3 oral sont recommandés avant un accouchement par césarienne urgent si l'on planifie une anesthésie générale. »

En outre, dans la section 7.3 Apport oral pendant le travail, il est désormais acceptable d'autoriser une femme en travail actif à ingérer des liquides clairs comme elle le souhaite. Bien que les opinions soient très variées, et que les données probantes appuyant un bienfait ou un effet nocif de l'ingestion d'aliments solides pour les parturientes en travail actif soient limitées, ${ }^{7,8}$ il faut en général décourager les parturientes d'ingérer des aliments solides pendant le travail actif. Cependant : «Contrairement aux aliments solides, les liquides clairs sont éliminés relativement rapidement de l'estomac pendant le travail. Ainsi, en règle générale, on devrait permettre aux parturientes d'ingérer des liquides clairs comme elles le souhaitent pendant le travail actif. Chaque établissement devrait élaborer des protocoles concernant l'absorption d'aliments solides et de liquides clairs par les femmes en travail actif. "

- Malgré nos progrès constants en tant que spécialité, il est indubitable que la prise en charge des voies aériennes, et plus particulièrement des voies aériennes difficiles, est encore à l'origine d'une importante proportion des événements indésirables liés à l'anesthésie. ' À ce jour, le Guide est resté muet concernant la prise en charge des voies aériennes. Le Comité a intégré une nouvelle section 5.2 Prise en charge des voies aériennes afin d'émettre quelques recommandations initiales. Cette section sera améliorée et étoffée au fur et à mesure des éditions futures du Guide. Ainsi : "La prise en charge des voies 
aériennes, particulièrement en cas de voies aériennes difficiles, contribue significativement à la morbidité et à la mortalité liées à l'anesthésie. Une prise en charge adaptée des patients dont l'intubation est difficile, de manière prévue ou imprévue, chez lesquels l'intubation a échoué, lorsqu'une ventilation au masque ou le positionnement d'un dispositif supraglottique pourrait être difficile, ou qui nécessitent des voies aériennes chirurgicales, est essentielle à la sécurité des patients. Cette prise en charge comprend, sans nécessairement s'y limiter, une évaluation adaptée des voies aériennes, du matériel (par ex. chariots pour les voies aériennes difficiles, vidéolaryngoscopes, bronchoscopes), une formation et des exercices de simulation appropriés, du personnel de soutien et le recours aux protocoles et listes de contrôle adéquats afin de prendre en charge au mieux des voies aériennes difficiles. La SCA ne recommande pas un protocole, un algorithme ou une liste de contrôle en particulier pour la prise en charge des voies aériennes difficiles ou lors d'un échec d'intubation, mais elle encourage fortement les lecteurs à se référer à l'Annexe 4 pour accéder aux publications les plus récentes sur le sujet. "

- Un dosage prudent, une attention aux détails et le recours systématique au monitorage neuromusculaire sont des composantes bien connues de la sécurité et des meilleures pratiques lors de l'utilisation de bloqueurs neuromusculaires. L'édition 2018 du Guide a ajouté la recommandation selon laquelle le monitorage « devrait » être utilisé. Il ne fait aucun doute que le monitorage est essentiel afin d'évaluer avec précision une récupération neuromusculaire satisfaisante. Selon le Comité, la recommandation devait être renforcée, et par conséquent : «Un monitorage neuromusculaire doit être utilisé lors de l'administration de bloqueurs neuromusculaires. » Selon moi, le monitorage quantitatif (objectif) (par ex., l'électromyographie, la kinémyographie, l'accélérométrie) est idéal et supérieur au monitorage qualitatif (subjectif) avec des stimulateurs de nerfs périphériques utilisant des mesures tactiles et visuelles. ${ }^{10-12}$ Pour l'instant toutefois, le Comité a décidé de simplement recommander l'utilisation de monitorage, laissant à la discrétion de l'anesthésiologiste le choix de monitorage, les moniteurs quantitatifs n'étant pas toujours disponibles. Les moniteurs des blocs neuromusculaires sont passés de la catégorie de matériel de monitorage « accessibles en exclusivité » à « requis » dans la section 5.5 Matériel de monitorage requis lors de l'utilisation de bloqueurs neuromusculaires.

- Des modifications importantes ont été apportées à la section 5.7 Dossiers. Il est essentiel de documenter tout événement imprévu ou indésirable survenant pendant les soins anesthésiques. Les systèmes d'enregistrement et de signalement des événements indésirables doivent occuper une place importante dans les programmes départementaux de qualité et de sécurité (voir section 2.1-4); les départements peuvent également décider de rapporter ces événements en se servant de programmes nationaux tel que le Système canadien de rapport des incidents anesthésiques de la SCA. ${ }^{13}$ En plus d'enregistrer les signes vitaux monitorés et les événements de routine, "Tous les autres soins et événements anesthésiques peropératoires pertinents, y compris les événements imprévus ou indésirables, devraient également être enregistrés. »

Les systèmes électroniques de gestion de l'information en anesthésie (SGIA) ont remplacé les dossiers anesthésiques manuscrits dans certains centres, là où les ressources nécessaires étaient disponibles. La littérature sur la sécurité des patients en anesthésie appuie fortement l'utilisation des SGIA. Le Comité a intégré des recommandations liées aux avantages potentiels des SGIA par rapport aux dossiers manuscrits. Toutefois, étant donné que les SGIA sont un outil relativement nouveau, ils ont besoin d'un ardent défenseur local qui travaillera avec les équipes des technologies de l'information et biomédicales pendant leur mise en œuvre et leur utilisation, et ils peuvent possiblement devenir une distraction. Les avantages potentiels de ces systèmes semblent clairement l'emporter sur tout écueil. ${ }^{14}$ Le contenu ajouté lit comme suit : "Ces recommandations s'appliquent tant aux dossiers anesthésiques manuscrits qu'à ceux créés via des systèmes électroniques de gestion de l'information en anesthésie (SGIA). À l'heure actuelle, il n'existe aucune norme de pratique indiquant quelles données supplémentaires (par ex. données d'analyse de gaz, de ventilation et respiratoires) potentiellement récoltées par un système de dossier de SGIA devraient faire partie du dossier de santé permanent d'un patient.

Il a été démontré que les SGIA pouvaient avoir des avantages potentiels par rapport aux dossiers rédigés à la main dans plusieurs domaines clés, notamment en augmentant la lisibilité, la précision et la fiabilité des dossiers anesthésiques, en créant des données interrogeables favorisant l'amélioration de la qualité, la communication des résultats et de la performance et la recherche translationnelle, une meilleure innocuité et un meilleur suivi des médicaments, ainsi qu'un suivi et un soutien à la décision clinique en temps réel pour les utilisateurs. La littérature sur la sécurité des patients en anesthésie appuie fortement l'utilisation des SGIA. Il est important de mentionner que les SGIA permettent de créer une base de données de patients longitudinale, de telle 
manière que les antécédents anesthésiques importants des patients sont facilement trouvables si l'on veut les passer en revue, ce qui devrait être considéré comme une propriété importante pour tout système. La SCA ne promeut ni n'endosse un fournisseur ou un produit en particulier, mais elle reconnaît les avantages potentiels d'un SGIA bien conçu et mis en œuvre par rapport à des dossiers complétés manuellement là où les ressources institutionnelles et du département d'anesthésie permettent d'envisager la mise en æuvre d'un SGIA. Dans l'idéal, ce système devrait être mis en ouvre et géré en coopération avec le département des technologies de l'information de l'établissement, et devrait permettre la communication avec les autres bases de données électroniques de patients de l'établissement dans la mesure du possible. "

Le Comité des normes est conscient que notre processus de mise au point de directive pourrait bénéficier d'une approche plus validée et systématique, en utilisant par exemple le cadre GRADE (Grading of Recommendations Assessment, Development and Evaluation - soit Cotation de l'examen, de la mise au point et de l'évaluation des recommandations) pour catégoriser la qualité des données probantes lors de la mise au point de nos recommandations. ${ }^{15}$ Nous sommes en train d'évaluer la façon dont nous mettons actuellement au point nos directives et cherchons assidûment un processus amélioré et davantage fondé sur les données probantes.

À l'heure actuelle, la SCA ne dispose pas d'un processus lui permettant d'évaluer de façon rigoureuse et de potentiellement endosser des directives de pratique de l'anesthésie créées par des personnes ou des groupes en dehors de la SCA. Nous examinons de près les avantages potentiels, pour les membres de la SCA, de mettre au point et en œuvre un tel processus.

Parmi les autres sujets envisagés par le Comité des normes pour révision dans les éditions futures du Guide, citons les ressources humaines en anesthésie (étant donné le manque d'anesthésiologistes), les politiques de congé et de retraite, et l'amélioration de la section 2.3 Aptitude à la pratique en ce qui touche à l'identification et au soutien des professionnels en anesthésie qui pourraient être confrontés à des difficultés personnelles ou de santé ou pourraient être handicapés. En coopération avec le Comité sur les équipes de soin en anesthésie, nous sommes en train de réviser en détail l'Annexe 5 - Exposé de principe sur les assistants en anesthésie. Nous avons l'intention de publier un document mis à jour dans un avenir proche.

Enfin, j'aimerais remercier personnellement les Dr David McKnight et Hilary Grocott pour leur aide et leur soutien inestimables du Guide pendant mon mandat de président du Comité des normes.

Conflicts of interest None declared.
Editorial responsibility This submission was submitted by Dr. Hilary P. Grocott, Editor-in-Chief, Canadian Journal of Anesthesia.

Conflit d'intérêt Aucun.

Responsabilité éditoriale Cet article a été traité par Dr Hilary P. Grocott, rédacteur en chef, Journal canadien d'anesthésie.

\section{References}

1. Dobson G, Chow L, Flexman A, et.al. Guidelines to the Practice of Anesthesia - Revised Edition 2020. Can J Anesth 2020; 67. DOI: https://doi.org/10.1007/s12630-019-01507-4.

2. Health Council of Canada. Understanding clinical practice guidelines. November 2012. Available from URL: https:// healthcouncilcanada.ca/files/CPG_Backgrounder_EN.pdf.pdf (accessed September 2019).

3. Dobson G, Chow L, Flexman A, et.al. Appendix 1 - Canadian Standards Association Standards for Medical Devices and Equipment; Guidelines to the Practice of Anesthesia - Revised Edition 2020. Available as Electronic Supplementary Material. Can J Anesth 2020; 67. DOI: https://doi.org/10.1007/s12630-01901507-4.

4. Dobson G, Chow L, Flexman A, et.al. Appendix 4 - Guidelines, Standards and other Official Statements, Guidelines to the Practice of Anesthesia - Revised Edition 2020. Available from URL: https://www.cas.ca/en/practice-resources/guidelines-toanesthesia.

5. Dobson G, Chow L, Flexman A, et al. Guidelines to the Practice of Anesthesia - Revised Edition 2019. Can J Anesth 2019; 66: 75-108.

6. Rosen D, Gamble J, Matava C; Canadian Pediatric Anesthesia Society Fasting Guidelines Working Group. Canadian Pediatric Anesthesia Society statement on clear fluid fasting for elective pediatric anesthesia. Can J Anesth 2019; 66: 991-2.

7. Singata M, Tranmer J, Gyte GM. Restricting oral fluid and food intake during labour. Cochrane Database Syst Rev 2013; 8: CD003930.

8. Ciardulli A, Saconne $G$, Anastasio H, Berghella V. Less restrictive food intake during labor in low risk singleton pregnancies: a systematic review and meta-analysis. Obstet Gynecol 2017; 129: 473-80.

9. Joffe AM, Aziz MF, Posner KL, Duggan LV, Mincer SL. Management of the difficult tracheal intubation: ASA closed claims analysis. Anesthesiology 2019. DOI: https://doi.org/10. 1097/ALN.0000000000002815.

10. Naguib M, Brull SJ, Kopman AF, et al. Consensus statement on the perioperative use of neuromuscular monitoring. Anesth Analg 2018; 127: 71-80

11. Checketts MR, Alladi R, Ferguson K, et al. Recommendations for standards of monitoring during anaesthesia and recovery 2015: Association of Anaesthetists of Great Britain and Ireland. Anaesthesia 2016; 71: 85-93.

12. Welliver $M$. Neuromuscular block monitoring has new opportunities coming. AANA J 2018; 86: 79-80.

13. Beattie WS, Culwick MD, Grocott HP. Canadian Anesthesia Incident Reporting System (CAIRS): The Canadian Anesthesiologists' Society's National Patient Safety Initiative. Can J Anesth 2018; 65: 749-56.

14. Deshur MA. Levine WC. AIMS: Should we AIM Higher? Anesthesia Patient Safety Foundation Newsletter 2015 June. Available from URL: https://www.apsf.org/apsf-newsletter/ (accessed September 2019). 
15. GRADE Working Group. The Grading of Recommendations Assessment, Development and Evaluation (GRADE). Available from URL: gradeworkinggroup.org (accessed September 2019).
Publisher's Note Springer Nature remains neutral with regard to jurisdictional claims in published maps and institutional affiliations. 